\title{
APPLICATION OF CUT ALGORITHM BASED ON ALGEBRAIC CONNECTIVITY TO COMMUNITY DETECTION
}

\author{
FUQIANG ZHAO*,*, LICHAO ZHANG*, GUIJUN YANG ${ }^{\dagger}$, \\ LI HE* and FENGYU YAN* \\ *Department of Information Science and Technology, \\ Tianjin University of Finance and Economics, \\ Tianjin 300222, P. R. China \\ †Department of Statistics, \\ Tianjin University of Finance and Economics, \\ Tianjin 300222, P. R. China \\ fqzhao@126.com
}

Received 8 September 2016

Revised 23 March 2017

Accepted 11 April 2017

Published 15 May 2017

\begin{abstract}
In the graph of a complex network, the algebraic connectivity is the second smallest eigenvalue of a Laplacian matrix. In this paper, we present a cut algorithm based on edge centrality by minimizing the algebraic connectivity of graph. The edge centrality cut algorithm (ECCA) cuts $k$ edges at a time in order to reduce temporal complexity, the algebraic connectivity of which experiences the fastest decline. To prevent nodes from overcutting, each edge sets the weight. We use the advanced ECCA (AECCA) to detect overlapping communities by calculating the correlation coefficients of the nodes. This paper also proposes upper, lower and weaker lower bounds of algebraic connectivity. We demonstrate that our algorithms are effective and accurate at discovering community structure in both artificial and real-world network data and that the algebraic connectivity of the cut algorithm lies between the upper and lower bounds. Our algorithms offer new insights into community detection by calculating the edge centrality.
\end{abstract}

Keywords: Algebraic connectivity; Laplacian matrix; cut edge; upper and lower bounds.

\section{Introduction}

Online social networks such as Twitter, Facebook, and Google+ contain a tremendous amount of data. With the rapid development of these networks in recent decades, there are likely millions or even billions of nodes [4]. The handling of massive

$\$$ Corresponding author.

This is an Open Access article published by World Scientific Publishing Company. It is distributed under the terms of the Creative Commons Attribution 4.0 (CC-BY) License. Further distribution of this work is permitted, provided the original work is properly cited. 
data contributes to the development of complex network models and methods. With the rise of mixed complex networks, it has become challenging to determine the number of complex networks in a community, and imbalance in communities emerges at the same time. The discovery of community structure in a complex network has increasing theoretical and practical significance. Researchers have developed a variety of methods to detect community structures. For instance, the traditional GirvanCNewman (GN) algorithm cuts an edge every time and has high time complexity: its running time is $O\left(m^{2} n\right)$ or $O\left(n^{3}\right)$ for a sparse network, where $n$ denotes the number of nodes and $m$ the number of edges. Radicchi et al. proposed an improved, self-contained version of the GN algorithm in [20], which is considerably faster than the traditional GN algorithm. The improved algorithm makes it possible to select the optimal division among all possible results produced by the algorithm [15]. Wilkinson, Huberman and Tyler proposed a method that only samples some nodes to calculate the edge-betweenness of the shortest path. This method increases computation speed at the cost of a reduction in accuracy. Chen and Yuan assumed that community divisions provided by the GN algorithm are typically unbalanced, as it considers all possible shortest paths between nodes when calculating edge betweenness. Accordingly, they proposed an advanced GN algorithm [5] that calculates edge betweenness based on the nonredundant shortest path between nodes on different sides. Fortunato et al. introduced a method to detect community structure that uses information centrality: edges between communities are those with the highest information centrality [13]. Although this advanced GN algorithm runs to completion in time $O\left(m^{3} n\right)$, or $O\left(n^{4}\right)$ for a sparse network, it is highly effective, particularly when the communities are very mixed. Some scholars have also proposed community cut models, but all the relevant algorithms appear to have high time complexity. The computation time of the algorithm proposed by Clauset et al. [9] is $O\left(n \log ^{2} n\right)$. The algorithm developed by Duch and Arenas [10] has a computation time of $O\left(n^{2} \log n\right)$. The algorithm of Eckmann and Moses [11] runs in $O\left(m\left\langle k^{2}\right\rangle\right)$. The algorithm proposed by Capocci et al. [3] leads to a total scaling of computation time as $O\left(n^{2}\right)$. Zhou and Lipowskys algorithm [24] runs in $O\left(n^{3}\right)$. An advanced algorithm should focus on two major aspects: how to improve the efficiency of the cut model, and how to reduce the time complexity of the algorithm to detect community structure in complex networks based on accurate results. In this paper, we present a weighted edge centrality cut algorithm (ECCA) and an advanced ECCA. Furthermore, we propose upper bounds, lower bounds, and weaker lower bounds on algebraic connectivity based on the idea of an added model [14].

\section{Problem Statement}

Let $G=(V, E)$ represent a complex network with $n$ nodes and $m$ edges, where $V$ denotes the set of nodes and $E$ the set of edges. We use edge $l$ to connect nodes $i$ and $j$, and define edge vector $a_{l} \in R^{n}$ as $a_{l i}=1, a_{l j}=-1$; all other entries are assumed to 
be 0 . The incidence matrix $A^{n \times m}$ of the graph $G$ is the matrix with the $l$ th column $a_{l}$. The Laplacian $L$ of $G$ is the following $n \times n$ matrix:

$$
L=A A^{T}=\sum_{i=1}^{m} a_{l} a_{l}^{T},
$$

where $L_{i i}$ is the degree of node $i$, and $L_{i j}=-1$ if $(i, j) \in E$, and is 0 otherwise.

In spectral graph theory, the Laplacian matrix can be used to analyze the characteristics of complex networks. All the following are properties belonging to the Laplacian:

Lemma 1. The Laplacian matrix is always positive-semidefinite: $L \geq 0$.

Lemma 2. The smallest eigenvalue of the Laplacian matrix is always 0 , and the corresponding eigenvector $v=[1,1,1 \cdots 1]$.

Lemma 3. The Laplacian matrix always has $n$ eigenvalues $0=\lambda_{1} \leq \lambda_{2} \leq \cdots \leq \lambda_{n}$.

Lemma 4. The number of times 0 appears as an eigenvalue in the Laplacian is the number of connected components in the graph.

The second smallest eigenvalue $\lambda_{2}(L)$ is called the algebraic connectivity of graph $G$, and the corresponding normalized eigenvector is called the Fiedler vector [12]. Several experiments have shown that algebraic connectivity is an effective measure of graph connectivity. The algebraic connectivity function of complex network $\lambda_{2}(L(x))$ is monotonic and convex. If two graphs $G_{1}=\left(V, E_{1}\right)$ and $G_{2}=\left(V, E_{2}\right)$ have the same vertex and their edges satisfy $E_{1} \subseteq E_{2}, \lambda_{2}\left(L_{1}\right) \leq \lambda_{2}\left(L_{2}\right)$. Thus, in a connected graph with the same vertex set, more edges indicate greater algebraic connectivity. The approximate value of $\lambda_{2}(L(x))$, can be computed by minimizing the second eigenvector of $L$ as follows [6-8]:

$$
\lambda_{2}(L)=\min _{\|v\|_{2} \dot{=}=1, v \perp 1} v^{T} L v .
$$

However, the time complexity is too high, and we can approximately calculate the algebraic connectivity function by the Gossip algorithm to effectively reduce it [1].

The second smallest eigenvalue of the Laplacian matrix $\lambda_{2}$ is directly associated with the structure of complex networks. We can regard $\lambda_{2}$ as a network connectivity function $\lambda_{2}(L(x))$. We can select an edge set that most quickly reduces network connectivity through an optimization model. The optimization problem is defined as follows:

$$
\begin{gathered}
\text { minimize } \quad \lambda_{2}(L(x)) . \\
\text { subject to } \quad 1^{T} x \leq k, \quad 0 \leq x \leq 1 .
\end{gathered}
$$

For the problem of the discovery of community structure in a complex network, the descriptions are as follows: Given an initial graph $G=(V, E)$, let $E_{\text {cut }}$ be the set of $k$ edges that most affect the algebraic connectivity when they are cut from $G$. 
We assume that $E_{\text {cut }} \subseteq E$. Then, (3) can be written as

$$
\begin{gathered}
\operatorname{minimize} \quad \lambda_{2}\left(L\left(E-E_{\text {cut }}\right)\right) \\
\text { subject to }\left|E_{\text {cut }}\right| \leq k, \quad E_{\text {cut }} \subseteq E .
\end{gathered}
$$

This process can be formulated as a Boolean problem. Every edge of $G$ can be encoded using a Boolean vector $x \in\{0,1\}^{m}$, where $x_{l}=1$ if edge $l \in E_{\text {cut }}$, and $x_{l}=0$ otherwise. Let $L$ denote the Laplacian matrix corresponding to $G$. Then (4) can be defined as (5), with variable $x$ :

$$
\begin{aligned}
\text { minimize } & \lambda_{2}\left(L-\sum_{l=1}^{m} x_{l} a_{l} a_{l}^{T}\right) \\
\text { subject to } & 1^{T} x \leq k, \quad x \in\{0,1\}^{m} .
\end{aligned}
$$

Let the constraint condition $x \in\{0,1\}^{m}$ be revised as $0 \leq x_{l} \leq 1$, which gives the variable a larger feasible set:

$$
\begin{aligned}
\text { minimize } & \lambda_{2}\left(L-\sum_{l=1}^{m} x_{l} a_{l} a_{l}^{T}\right) \\
\text { subject to } & 1^{T} x \leq k, \quad x \in[0,1]^{m}
\end{aligned}
$$

and (6) is the lower bound on (5).

When a complex network is cut into two unconnected subgraphs, the iteration terminates. However, the degree distribution of the nodes obeys the power-law form in social network. A large number of nodes have degrees less than $k$. These can be peeled off when we delete the edges of the relevant node. We then obtain two unconnected subgraphs, which are asymmetric. This operation has a negative influence on the discovery of community structure in a complex network. For this reason, every edge in the graph is weighted in our algorithm. The weight $w_{l}$ is higher if the nodes are in the same community and lower if they are from different communities. The weight is defined as follows:

$$
w_{l}=\frac{1}{e^{\frac{\left|f_{i}+f_{j}\right|}{2}}},
$$

where $l \sim(i, j)$, and $f_{i}$ and $f_{j}$ are the $i$ th and $j$ th components of the Fiedler vector, respectively. This approach avoids forming two asymmetrical disconnected subgraphs when an edge is cut improperly in the process of community discovery.

The components of the Fiedler vector, which is obtained by solving the Laplacian matrix, are both positive and negative. Equation (7) represents the sum of two components. The weight approximates 1 , and cannot yield the advantages of setting a weight when the absolute values of two components are similar and have different signs. In this manner, (7) should be modified as follows:

$$
w_{l}=\frac{1}{e^{\frac{\left|f_{i}\right|+\left|f_{j}\right|}{2}}} .
$$


In general, the selection of a weight affects the performance of the algorithm, and we should define the weight in the algorithm well. In a community network, the nodes with more nodes adjacent to them are likely to connect nodes in different communities. Based on the equation $f_{i}=\frac{-f_{e}-\cdots-f_{i}-\cdots-f_{k}}{\lambda_{2}-d_{n}}$, the values of the Fiedler vector corresponding to the nodes are smaller when the degrees of the nodes are greater, or when the adjacent nodes in the same community are distributed uniformly in a different community. Thus, the weight defined in (8) can be improved as follows:

$$
w_{l}=\frac{1}{e^{\max \left(\left|f_{i}\right|,\left|f_{j}\right|\right)}} .
$$

Then, (6) can be defined as (10), which considers weight $w_{l}$ :

$$
\begin{gathered}
\operatorname{minimize} \quad \lambda_{2}\left(L-\sum_{l=1}^{m} x_{l} \frac{1}{e^{\max \left(\left|f_{i}\right|,\left|f_{j}\right|\right)}} a_{l} a_{l}^{T}\right) \\
\text { subject to } 1^{T} x \leq k, \quad x \in[0,1]^{m} .
\end{gathered}
$$

\section{Edge Centrality Cut Algorithm}

In this section, we describe the edge centrality cut algorithm for solving (10). We provide the following definitions that are used in our algorithm:

Definition 1. The edge that leads to the greatest reduction in algebraic connectivity is defined as the center edge. This property of the edge is called edge centrality.

Definition 2. The second smallest eigenvalue of the Laplacian matrix is called algebraic connectivity function. It measures the connectivity of a graph.

In contrast to the traditional algorithms used to detect communities, the ECCA defines edge centrality by spectral analysis. In contrast to algorithms that use the shortest distance and random walking, this cut algorithm is better for medium-sized networks with higher efficiency and accuracy. It deletes the node with the highest spectral centrality and recalculates the spectral centrality of the remaining nodes. This cut algorithm is as follows:

(1) Calculate the spectral centrality for each edge of graph $G$ based on the edge centrality function.

(2) Find $k$ edges with the highest spectral centrality $(k \geq 1)$, and delete them; renew the complex network to $G^{\text {new }}$. The choice of $k$ is based on the degree of edge sparseness of complex networks.

(3) Calculate the algebraic connectivity of the graph. If $\lambda_{2}=0$, go to step 4; otherwise return to step 1.

(4) Update the new complex network graphs $G_{1}^{\text {new }}$ and $G_{2}^{\text {new }}$. The graph $G$ is divided into two communities. 
In this algorithm, spectral centrality is measured based on the algebraic connectivity function. The gradient of the algebraic connectivity function $\lambda_{2}(L-$ $\left.w_{l} x_{l} a_{l} a_{l}^{T}\right)$ is $w_{l}\left(v_{i}-v_{j}\right)^{2}$. Let $v$ denote the Fiedler vector of $L, v_{i}$ is the $i$ th element of vector $v$.

\section{Upper and Lower Bounds on a Cut Algorithm}

In this section, we derive upper and lower bounds on the algebraic connectivity of a graph through cutting only one edge (unweighted cutting algorithm). Let $L$ be the Laplacian of the initial graph $G$. We assume that $\lambda_{2}(L)$ is isolated, i.e., $\lambda_{1}<\lambda_{2}<\lambda_{3}$, and the corresponding eigenvector $\mathrm{V}$ is normalized. When we cut the edge $l \sim(i, j)$ whose vector is $a_{l}$, we can obtain the following bounds for $\lambda_{2}\left(L-a_{l} a_{l}^{T}\right)$ :

Upper bound:

$$
\lambda_{2}\left(L-a_{l} a_{l}^{T}\right)<\lambda_{2}-\frac{\left(v_{i}-v_{j}\right)^{2}}{\frac{3}{2}+\frac{6}{\lambda_{2}-\lambda_{3}}} .
$$

Lower bound:

$$
\lambda_{2}\left(L-a_{l} a_{l}^{T}\right)>\lambda_{2}-\frac{\left(v_{i}-v_{j}\right)^{2}}{1+\sum_{i=3}^{n} \frac{u_{i}^{2}}{\lambda_{2}-\lambda_{i}}} .
$$

Since $\left(v_{i}-v_{j}\right)^{2}$ is a subgradient of $\lambda_{2}(L)$, we can obtain a weaker lower bound:

$$
\lambda_{2}\left(L-a_{l} a_{l}^{T}\right) \geq \lambda_{2}(L)-\left(v_{i}-v_{j}\right)^{2} .
$$

\subsection{Proof of the upper bound}

Let $L=Q \Lambda Q^{T}$, where $Q$ is the matrix of orthonormalized eigenvectors and $\Lambda$ is the diagonal matrix of eigenvalues. The matrices $L$ and $L-a_{l} a_{l}^{T}$ both have eigenvalue 0 , with corresponding eigenvector 1 . The remaining $n-1$ eigenvalues of $L-a_{l} a_{l}^{T}$ satisfy the following equation (see Fig. 1) [2, 16]:

$$
\sum_{i=2}^{n} \frac{u_{i}^{2}}{\lambda_{i}-d}=1
$$

where $u=Q^{T} a_{l}\left(u_{2}=v_{i}-v_{j}, u_{1}=0\right)$, and $d_{1}, d_{2}, d_{3}, \ldots, d_{n}$ denote the ordered eigenvalues of $L-a_{l} a_{l}^{T}$. According to the eigenvalue intersectionality, we have $d_{1}=\lambda_{1}<d_{2}<\lambda_{2}<\cdots<d_{n}<\lambda_{n}$.

Now, consider $d$ to be in the interval $\left(\lambda_{1}, \lambda_{2}\right)$. As $d_{i}$ are the roots of equation $\sum_{i=2}^{n} \frac{u_{i}^{2}}{\lambda_{i}-d}=1$, and $\sum_{i=2}^{n} \frac{u_{i}^{2}}{\lambda_{i}-d}=1$ can be seen as an increasing function of $d$, we have $d \geq d_{2}$ if $\sum_{i=2}^{n} \frac{u_{i}^{2}}{\lambda_{i}-d} \geq 1$, which means that $\frac{u_{2}^{2}}{\lambda_{2}-d} \geq 1+\sum_{i=3}^{n} \frac{u_{i}^{2}}{d-\lambda_{i}}$. Since $\|u\|=2$, $\sum_{i=3}^{n} u_{i}^{2} \leq 2$ for $\lambda_{i} \geq \lambda_{3}(i \geq 3)$. Thus, $\frac{u_{i}^{2}}{d-\lambda_{i}} \geq \frac{u_{i}^{2}}{d-\lambda_{3}}$, and $\sum_{i=3}^{n} \frac{u_{i}^{2}}{d-\lambda_{i}} \geq \frac{2}{d-\lambda_{3}}$. So if 


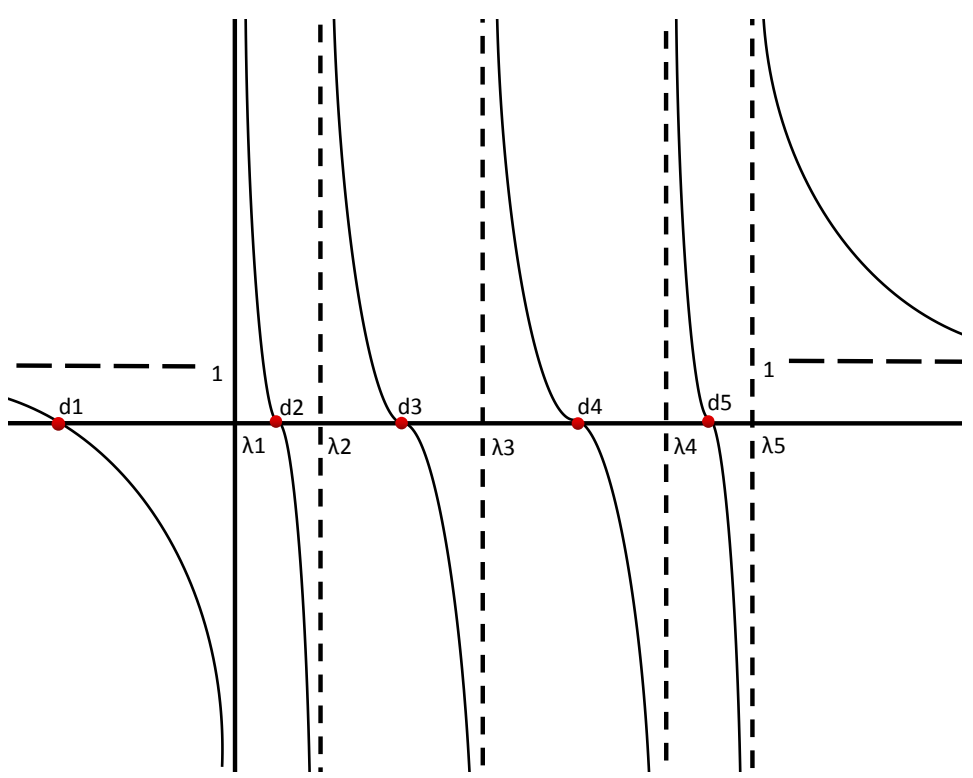

Fig. 1. Graph of Eq. (10), $n=5, \rho<0$.

$\frac{u_{2}^{2}}{\lambda_{2}-d} \geq 1+\frac{2}{d-\lambda_{3}}$, then $d \geq d_{2}$. Setting $\lambda_{2}-d=\varepsilon, \lambda_{2}-\lambda_{3}=\delta$. We want to find $\varepsilon>0$ such that

$$
\frac{u_{2}^{2}}{\varepsilon} \geq 1+\frac{2}{\delta-\varepsilon}
$$

It can be verified that setting

$$
\varepsilon=\frac{u_{2}^{2}}{2 \times\left(\frac{1}{\delta}+\frac{1}{4}+\sqrt{\frac{1}{4}+\frac{4}{\delta^{2}}}\right)},
$$

satisfies (15). Since $d \geq d_{2}$ and $\lambda_{2}-\varepsilon=d, d_{2} \leq \lambda_{2}-\varepsilon$. That is $d_{2} \leq \lambda_{2}-$ $\frac{u_{2}^{2}}{2 \times\left(\frac{1}{\delta}+\frac{1}{4}+\sqrt{\frac{1}{4}+\frac{4}{\delta^{2}}}\right)}$. Since $\sqrt{\frac{1}{4}+\frac{4}{\delta_{2}}} \leq \frac{1}{2}+\frac{2}{\delta}$, it is easy to verify that $d_{2} \leq \lambda_{2}-\frac{u_{2}^{2}}{\frac{3}{2}+\frac{6}{\delta}}$. Using $u_{2}=v_{i}-v_{j}$ and $\delta=\lambda_{2}-\lambda_{3}$, we obtain the upper bound in (11).

\subsection{Proof of the lower bound} Consider the function $\sum_{i=2}^{n} \frac{u_{i}^{2}}{\lambda_{i}-d}=1$; then, $d=\lambda_{2}-\frac{u_{2}^{2}}{1+\sum_{i=3}^{n} \frac{u_{i}^{2}}{d-\lambda_{i}}}$. Since $d_{i}$ satisfies
$\sum_{i=2}^{n} \frac{u_{i}^{2}}{\lambda_{i}-d}=1$, we have

$$
d_{2}=\lambda_{2}-\frac{u_{2}^{2}}{1+\sum_{i=3}^{n} \frac{u_{i}^{2}}{d_{2}-\lambda_{i}}}
$$


Using $d_{2} \leq \lambda_{2}$, we have $d_{2}-\lambda_{i} \leq \lambda_{2}-\lambda_{i}<0$, and

$$
\lambda_{2}-\frac{u_{2}^{2}}{1+\sum_{i=3}^{n} \frac{u_{i}^{2}}{d_{2}-\lambda_{i}}}>\lambda_{2}-\frac{u_{2}^{2}}{1+\sum_{i=3}^{n} \frac{u_{i}^{2}}{\lambda_{2}-\lambda_{i}}} .
$$

That is, $d_{2}>\lambda_{2}-\frac{\left(v_{i}-v_{j}\right)^{2}}{1+\sum_{i=3}^{n} \frac{u_{i}^{2}}{\lambda_{2}-\lambda_{i}}}$. Thus, we obtain the lower bound in (12).

\section{Experiments}

To verify our improved algorithms, we do experiments on real world networks and synthetic benchmark networks. Our experiments are done in the same hardware and running environment: double CPU, Windows7 64bit, with a memory of $4 \mathrm{G}$.

\subsection{Tests on real world networks}

\subsubsection{Zachary's Karate club network}

The real network was Zacharys network of Karate club members, a well-known graph regularly used as a benchmark to test community detection algorithms. It consisted of 34 vertices and 78 edges, the members of a karate club in the United States who were observed during a period of three years. The result of ECCA was the same as that for the Laplacian matrix on the karate network. Compared to results obtained using the real-world network, the results are shown in Fig. 2.

Newman and Girvan [19] proposed the modularity to measure the strength of the community structure found by algorithms. Thus, we can obtain the modularity value by

$$
Q=\sum_{i} e_{i j}-a_{i}^{2}=\operatorname{Tr}(e)-\left\|e^{2}\right\| .
$$

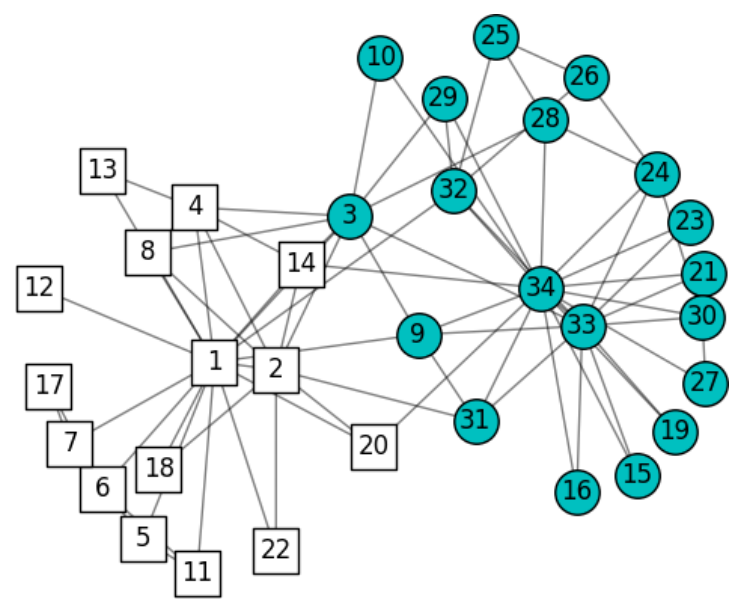

Fig. 2. Zachary's Karate Club Network bipartition found by ECCA. 
Table 1. Comparisons among the modularity values for different cut algorithms.

\begin{tabular}{lccc}
\hline Dataset & GN & ECCA $(k=1)$ & AECCA $(k=1)$ \\
\hline Karate & 0.3850 & 0.3863 & 0.4020 \\
Football & 0.4270 & 0.4559 & 0.5032 \\
\hline
\end{tabular}

We define a $k \times k$ symmetric matrix $e$ whose element $e_{i j}$ is the fraction of all edges in the network that link vertices in community $i$ to vertices in community $j$. Where $a_{i}=\sum_{i} e_{i j}, \operatorname{Tr}(e)=\sum_{i} e_{i i}$ and $\|x\|$ indicates the sum of the elements of the matrix $x$.

We compare GN with ECCA and AECCA (Advanced ECCA, Sec. 5.5) based on the modularity. The results are also shown in Table 1. We note that ECCA and AECCA are generally better than GN in terms of modularity.

\subsubsection{American college football network}

We test cut algorithm with different values of $k$ on the American College Football Network. The network contained 115 nodes and 613 edges. As shown in Fig. 3, $\lambda_{2}$ was zero after three iterations by the ECCA method with $k=30$, and the network was divided successfully in the shortest number of iterations compared to ECCA with other values of $k$. A larger $k$ gives a less number of iterations. However, a higher value of $k$ may lead to excessive segmentation of a complex network. As shown in Table 2, Different $k$ values influenced the modularity of the method. As $k$ increases, the modularity of cut algorithm decreases.

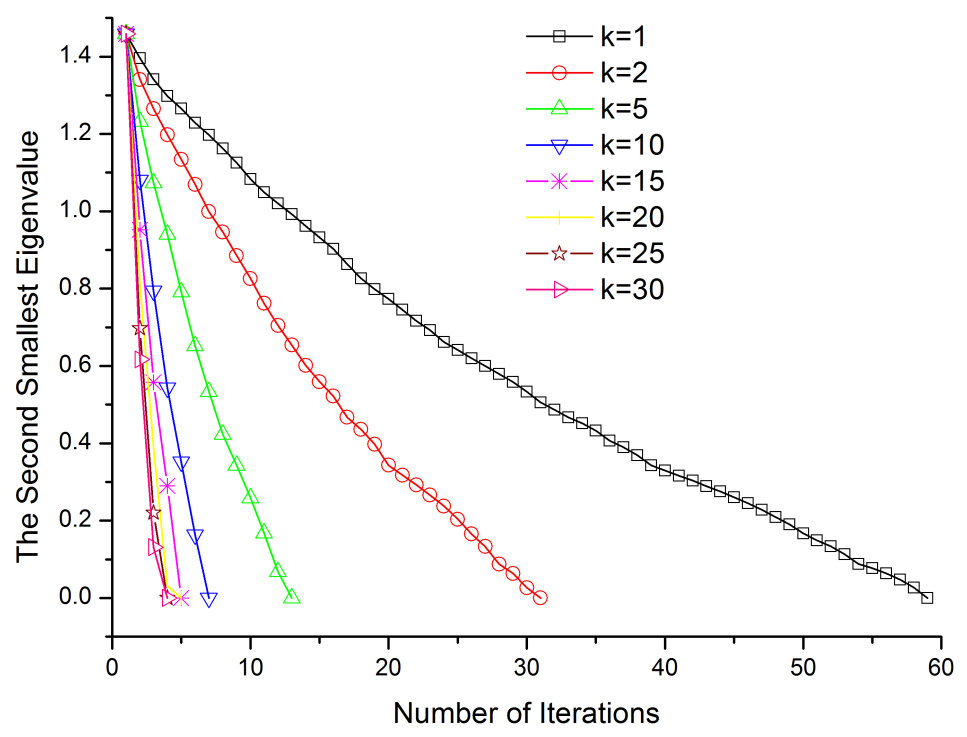

Fig. 3. The second smallest eigenvalue with different values of $k$. 
Table 2. Modularity results for ECCA with different values of $k$.

\begin{tabular}{cccccc}
\hline & $k=1$ & $k=2$ & $k=5$ & $k=10$ & $k=20$ \\
\hline$Q$ & 0.4559 & 0.5147 & 0.5082 & 0.5011 & 0.4343 \\
\hline
\end{tabular}

We also use the random cutting edge method, the GN method, and the ECCA method to delete $k$ edges randomly, delete $k$ edges with the highest betweenness, and delete $k$ edges with the highest spectral centrality, respectively. The initial value of $\lambda_{2}$ for the American College football network was 1.459001. ECCA yielded the same result as the GN and random methods, but with higher efficiency.

\subsection{Tests on artificial networks}

We utilize the benchmark proposed by Lancichinetti et al. in [17]. Many parameters are used to control the generated networks: the number of nodes $N$, the average node degree $k n$, the maximum node degree $\max k$, the mixing parameter $\mu$, the minimum community size $\min c$, and the maximum community size $\max c$, where $N=500$, $k n=15, \max k=50, \min c=20$, and $\max c=50$. A larger $\mu$ gives a fuzzier community structure. In addition, we adopt the normalized mutual information (NMI) to compare the partition found by community detection methods against the true partition. A larger NMI indicates a better method.

As shown in Fig. 4, both ECCA and GN are effective at identifying the correct number of communities when the mixing parameter $\mu$ is smaller than 0.25 . However,

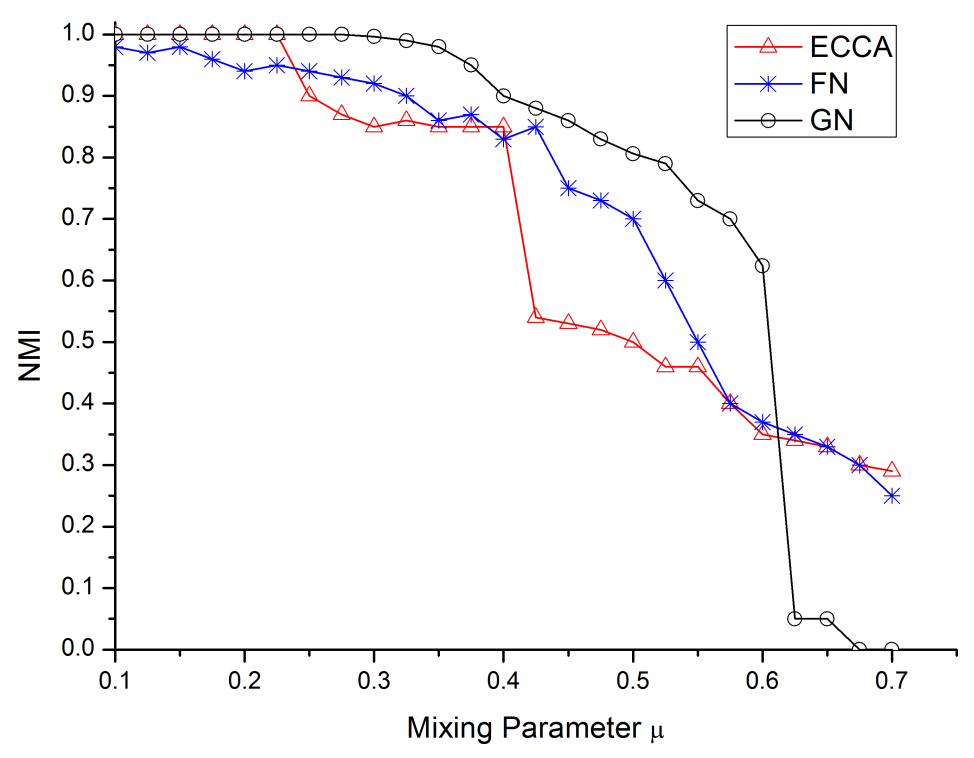

Fig. 4. NMI as a function of the mixing parameter of the benchmark graphs. 


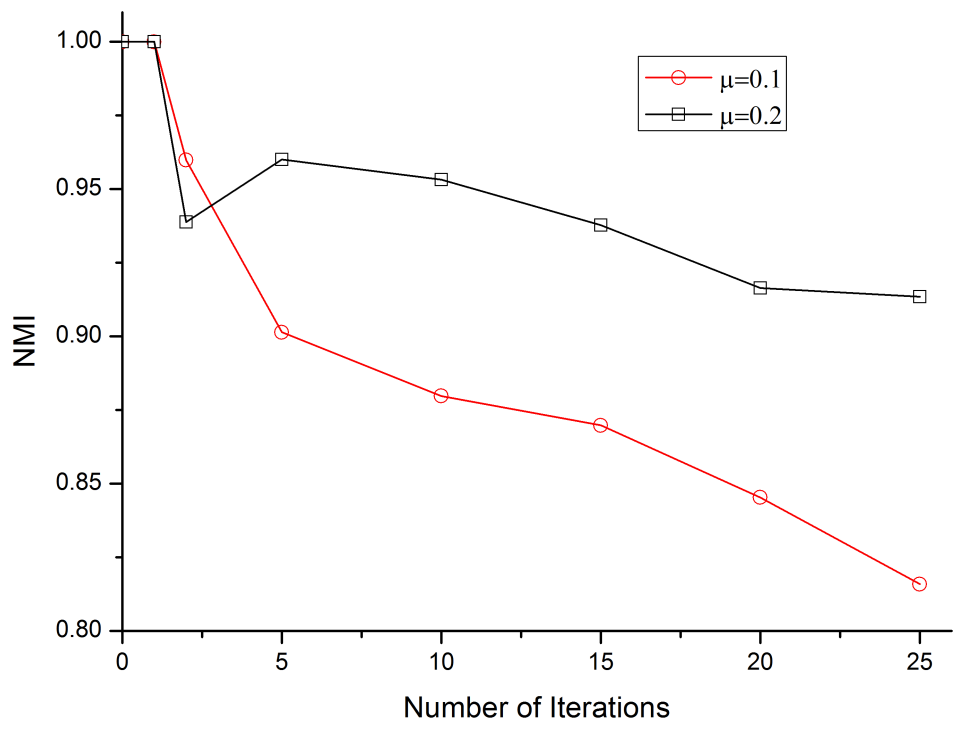

Fig. 5. NMI results for different mixing parameters $\mu$.

when the community structure becomes fuzzier with an increased $\mu$, the performance of ECCA deteriorates. GN and Fast Newman (FN) outperform ECCA, when the mixing ratio $\mu$ is larger than 0.4. As mixing parameter increases, the NMIs of all algorithms decrease, especially when mixing parameter $\mu$ is bigger than 0.5 , NMI suddenly drops.

By tuning $k$, we test the effectiveness of our method on networks with different fuzziness of communities. As shown in Fig. 5, our model has an excellent module identification performance, when the $k$ has values less than 5 . When $k$ is greater than 5 , NMI gradually decreases under higher mixing parameter $(\mu=0.2)$.

\subsection{Analysis of upper and lower bounds of cut algorithm}

To test the accuracy of the bounds we deduced, we applied them to a dolphin network [18]. The results are shown in Fig. 6 (The horizontal axis represents the number of edges cut and the vertical axis the second smallest eigenvalue $\lambda_{2}$ ). The value of $\lambda_{2}$ was zero following the deletion of six edges, and the network was divided into two parts. The value of $\lambda_{2}$ obtained by ECCA was between the lower and upper bounds. The black dotted line represents (snow) the upper bound on the algebraic connectivity of the cut algorithm, the blue line represents (plus) the lower bound, and the green dotted line represents (circle) the weaker lower bound on the algebraic connectivity of the cut algorithm. The red line represents (triangle) the value of $\lambda_{2}$ obtained by ECCA.

Figure 7 shows the change in the algebraic connectivity of the American College Football Network after 20 edges were deleted. When cutting 20 edges along the 


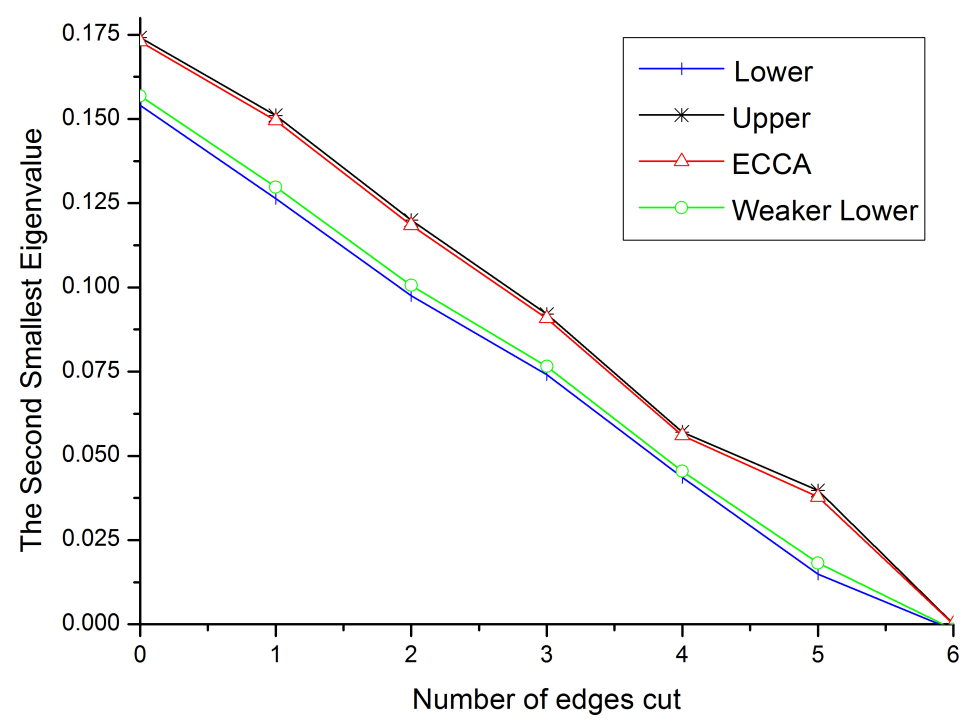

Fig. 6. (Color online) Bounds on the algebraic connectivity of dolphin network.

horizontal axis, the vertical axis was reduced to 0.6 , and $\lambda_{2}$ became zero after 58 edges were deleted. It is noted that the values of $\lambda_{2}$ is very close to the upper bound and relatively far from the lower bound, therefore, a tighter lower bound is worthy of further study.

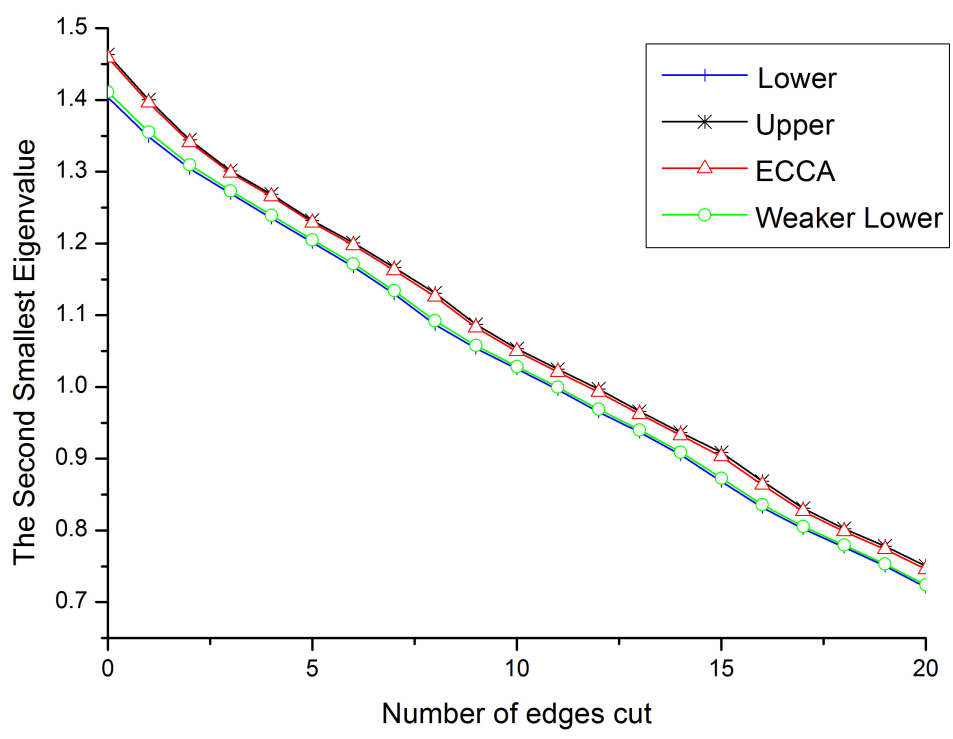

Fig. 7. Bounds on the algebraic connectivity of college football network. 


\subsection{Analysis of time complexity}

The traditional GN algorithm deletes the edge with the highest edge betweenness in a single iteration each time. The iterative algorithm runs in $O\left(n^{2} m\right)$ time on a network with $n$ nodes and $m$ edges. Compared to the GN algorithm, the spectrum optimization algorithm deletes $k$ edges by the cutting edge learning model and calculates the eigenvector (Fiedler vector) corresponding to the second smallest eigenvalue of the Laplacian in a single iteration each time, until the complex network is divided into two unconnected subgraphs. This process can be completed in time $O\left(m_{1} \times n\right)$, where $m_{1}$ is the number of iterations, typically greater than 100. This algorithm requires storage for an $m_{1}$ Lanczos vector with $n$ dimensions. Its shortcoming is its high spatial complexity. The calculation of the spectral centrality of all edges based on the Fiedler vector then takes $O(m)$ time. The time complexity of deleting the edge with the highest spectral centrality is $O(m)$. The algebraic connectivity function $\lambda_{2}\left(L\left(G^{\text {new }}\right)\right)$ is applied to calculate the algebraic connectivity of the updated network. This process is based on the Gossip algorithm, and can be completed in time $O\left(m_{2} \times \log (n)\right)$, where $m_{2}$ is the number of iterations and should be a constant. Each time the cutting edge learning model is executed, the time complexity is $O\left(m_{1} \times n+2 m+m_{2} \times \log (n)+n\right) \rightarrow O\left(m_{1} n+m\right)$. When the complex network is divided into two unconnected subgraphs, the algorithm is applied to each subgraph recursively. If the algorithm is executed on each subgraph, its efficiency can drastically improve.

\subsection{ECCA with correlation coefficients}

The Laplacian matrix and the modular matrix were applied to community detection in [21-23]. The eigenvalues of the Laplacian matrix and the modular matrix reflect the significance of each eigenvector. The gap between any two eigenvalues, resulting from the sorting of eigenvalues, can provide important information for multi-scale community detection. A larger gap indicates a greater difference in significance. We calculated the Laplacian matrix and the modularity matrix of Zachary's Karate Club followed by their eigenvalues. The real network was Zacharys network of Karate club members, a well-known graph regularly used as a benchmark to test community detection algorithms. It consisted of 34 vertices and 78 edges, the members of a Karate club in the United States who were observed during a period of three years. The results are shown in the figures below.

Figure 8 shows the eigenvalues of the Laplacian matrix. Figure 9 shows the eigenvalues of the modular matrix. The horizontal axis shows the eigenvalues of matrix and the vertical axis represents the rank index of eigenvalues in descending order. As shown in Fig. 8, the largest eigengap of the Laplacian matrix occurs between the first and second smallest eigenvalues. It indicates that it is appropriate to utilize only the second eigenvector (the Fiedler's vector) and the number of communities is 2 . 


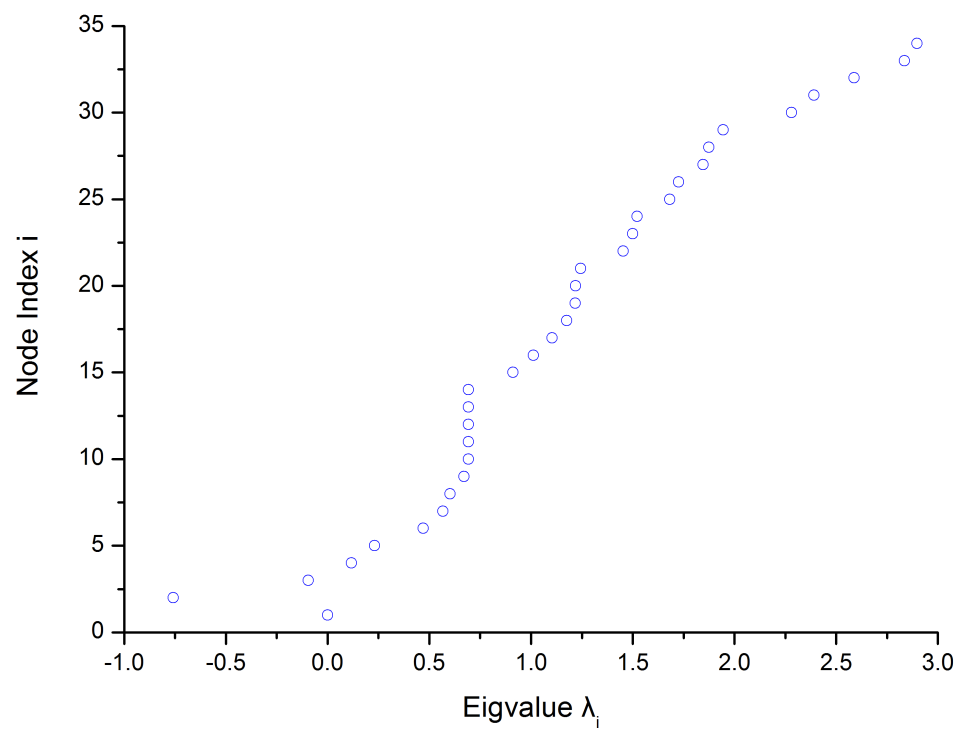

Fig. 8. The eigenvalue of the Laplacian matrix.

Figure 9 shows that the largest eigengap of the modularity matrix resides between the largest and the second largest eigenvalues. This indicates that only the Fiedler's vector is the significant eigenvector and it partitions the network into two groups. The other eigengap occurs between the second and third largest eigenvalues for the

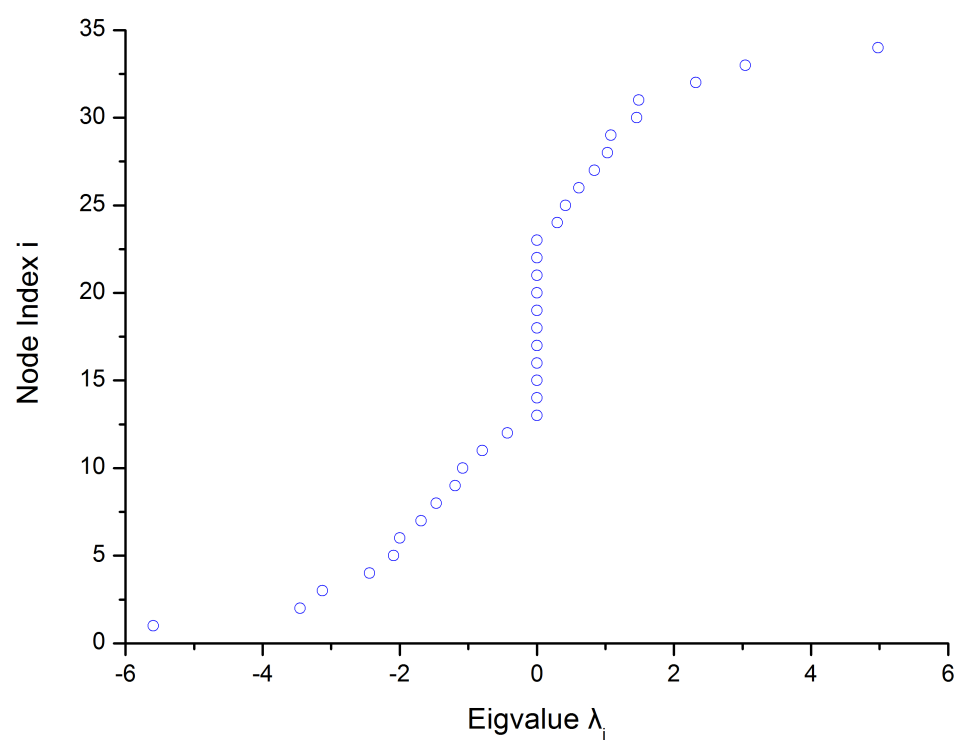

Fig. 9. The eigenvalue of the modular matrix. 


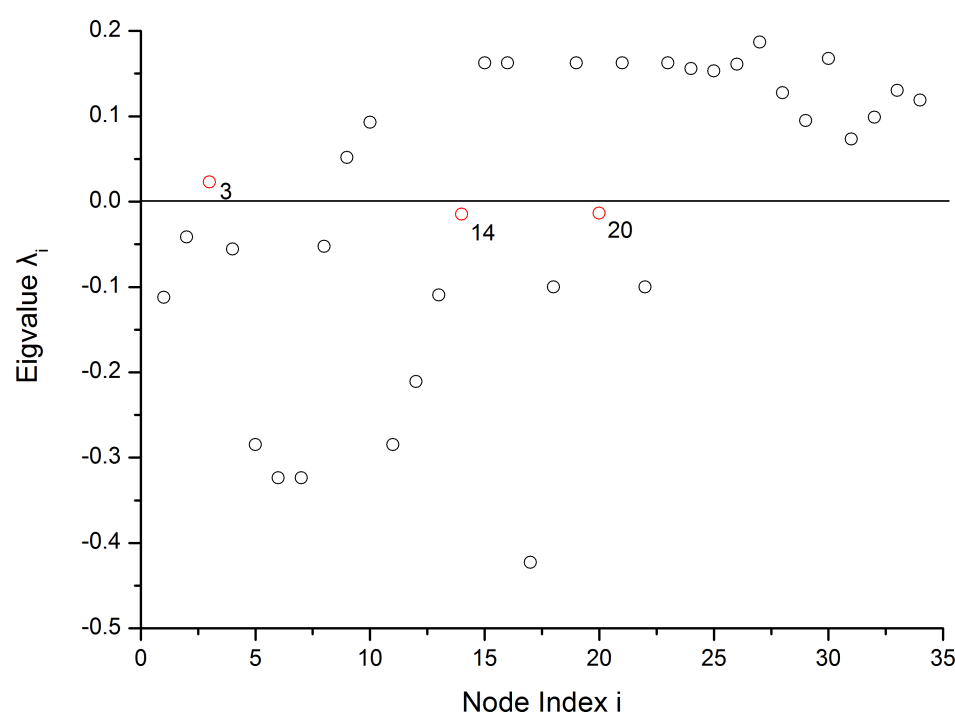

Fig. 10. Fielder vector of Zachary's Karate Club network.

modularity matrix. This eigengap indicates the other significant topological scale corresponds to the partition dividing the network nodes into three communities. The same method could have been used in the problem of the eigengap between the third largest and the fourth largest eigenvalues.

The result of ECCA was the same as that for the Laplacian matrix on the Karate network. Compared to results obtained using the real-world network, the results are shown in Fig. 2.

As shown in Fig. 10, the eigenvalues of nodes 3, 14, and 20 were close to zero. It was difficult to identify the communities to which nodes 3,14 , and 20 belonged. The absolute value of the difference between the eigenvalues of these three nodes and 0 was less than the threshold value alpha. However, the difference in the number of edges of nodes 14 and 20 that connect to the two communities is not less than 2 . Therefore, the correlation coefficient between node 3 (overlapping community node) and its neighbor nodes was calculated. Node 3 should have been in the same community as nodes 0 and 1 .

The results of community detection by advanced ECCA are shown in Fig. 11. The modularity method was the same as for the actual network. Hence, the advanced ECCA is as follows:

(1) Calculate spectral centrality for each edge of graph $G$ based on the edge centrality function, and sort them.

(2) Find $k$ edges with the highest spectral centrality $(k \geq 1)$, and delete them; renew the complex network to $G^{\text {new }}$. The choice of $k$ is based on the degree of edge sparseness of complex networks. 


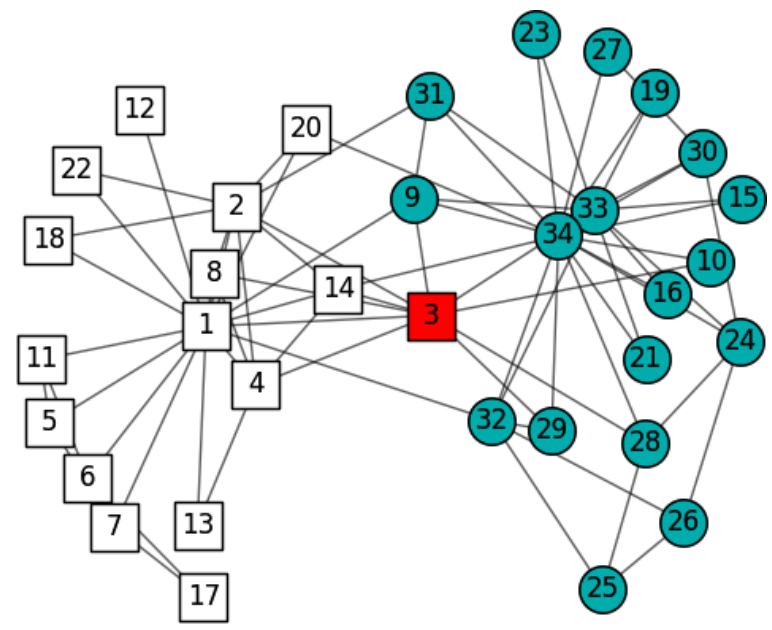

Fig. 11. Zachary's Karate Club Network bipartition found by AECCA.

(3) Calculate the algebraic connectivity of the graph. If $\lambda_{2}=0$, do to Step 4; otherwise, go to Step 1.

(4) When the corresponding component value of the Fiedler vector of a node is less than threshold $\alpha$, analyze the necessity of computing the correlation coefficient. Moreover, calculate the correlation coefficient of the node if the difference in the number of edges that it connects to the two communities is less than 2 .

(5) Use (20) to calculate the correlation. By comparing the correlation coefficients of the nodes in the two communities, we can determine the community to which the node belongs.

(6) Update the new complex network graphs $G_{1}^{\text {new }}$ and $G_{2}^{\text {new }}$. Graph $G$ has been divided into two communities:

$$
r_{i j}=\frac{\left\langle x_{i}, x_{j}\right\rangle-\left\langle x_{i}\right\rangle\left\langle x_{j}\right\rangle}{\left[\left(\left\langle x_{i}^{2}\right\rangle-\left\langle x_{i}\right\rangle^{2}\right)\left(\left\langle x_{j}^{2}\right\rangle-\left\langle x_{j}\right\rangle^{2}\right)\right]^{\frac{1}{2}}},
$$

where average $\langle$.$\rangle is over the first few nontrivial eigenvectors, r_{i j}$ measures community closeness between nodes $i$ and $j . x_{i}$ and $x_{j}$ are the $i$ th and $j$ th components of the Fiedler vector, respectively.

\section{Conclusion}

The network is dichotomized when the second smallest eigenvalue of the Laplacian matrix, which determines the algebraic connectivity of a complex community, is zero. The time complexity of the traditional cut algorithm is high. To address this, we proposed here a novel cut algorithm based on a centrality measure. Our algorithm deletes edges with the greatest implications for the algebraic connectivity of the 
network. At the same time, the calculation of edge weights is used in the community division, and the problem of excessive segmentation of the network should be avoided. Experiments on both real and artificial networks showed that our cut algorithm can reduce time complexity, and its results are consistent with those of the GN algorithm. We also deduced the upper bound, the lower bound, and a weaker lower bound on the algebraic connectivity of a complex network by analyzing the cut algorithms. Several experiments were used to verify our result.

A number of extensions or improvements to our algorithms are possible and worthy of further investigation. First, we hope to study whether these algorithms are suitable for large-scale complex networks and the lower bound of algebraic connectivity is improved to be tighter. Second, we hope to verify whether they can reduce time complexity further by cutting several edges each time. Finally, we hope to realize the integrated application of cut algorithms and add algorithms, i.e., by adding edges to a network in addition to cutting edges, to a certain extent, to detect communities effectively.

\section{Acknowledgments}

In this paper, the research was sponsored by the Natural Science Foundation of China (Project Nos. 11471239 and 61502331), Tianjin Natural Science Foundation of China (Project No. 15JCYBJC16000) and Tianjin Philosophy and Social Science Research Program Foundation Project China (Project No. TJTJ15-002).

\section{References}

[1] Boyd, S., Ghosh, A., Prabhakar, B. and Shah, D., Gossip algorithms: Design, analysis and applications, J. Proc. IEEE Infocom 3 (2005) 1653-1664.

[2] Bunch, J., Nielsen, C. and Sorensen, D., Rank one modification of the symmetric eigenproblem, J. Numer. Math. 31 (1978) 31-48.

[3] Capocci, A., Servedio, V. D. P., Caldarelli, G. and Colaiori, F., Detecting communities in large networks, J. Phys. A 352 (2005) 669-676.

[4] Aggarwal, C. C., Social Network Data Analytics (Springer Science + Business Media, LLC, 2011).

[5] Chen, J. and Yuan, B., Detecting functional modules in the yeast proteinCprotein interaction network, J. Bioinform. 22(18) (2006) 2283-2290.

[6] Chen, P.-Y. and Hero, A. O., Local fiedler vector centrality for detection of deep and overlapping communities in networks, IEEE Int. Conf. Acoustics, Speech and Signal Processing (2014), pp. 1120-1124.

[7] Chen, P.-Y. and Hero, A. O., Deep community detection, IEEE Trans. Signal Process. 63 (2014) 5706-5719.

[8] Chen, P.-Y. and Hero, A. O., Phase transitions in spectral community detection, IEEE Trans. Signal Process. 63(16) (2015) 4339-4347.

[9] Clauset, A., Newman, M. E. J. and Moore, C., Finding community structure in very large networks, Phys. Rev. E 70(6) (2004) 066111. 
[10] Duch, J. and Arenas, A., Community detection in complex networks using extremal optimization, J. Phys. Rev. E 70(2) (2005) 027104.

[11] Eckmann, J.-P. and Moses, E., Curvature of co-links uncovers hidden thematic layers in the World Wide Web, Proc. Natl. Acad. Sci. 99 (2002) 5825-5829.

[12] Fiedler, M., Algebraic connectivity of graphs, J. Czechoslovak Math. J. 23 (1973) 298-305.

[13] Fortunato, S., Latora, V. and Marchiori, M., Method to find community structures based on information centrality, Phys. Rev. E 70(5) (2004) 056104.

[14] Ghosh, A. and Boyd, S., Growing well-connected graphs, J. Proc. 45th IEEE Conf. Decision and Control (2006), pp. 6605-6611.

[15] Girvan, M. and Newman, M. E. J., Community structure in social and biological networks, J. Proc. Nat. Acad. Sci. USA 99(12) (2002) 7821-7826.

[16] Golub, G., Some modified matrix eigenvalue problems, J. SIAM Rev. 15 (1973) 318-334.

[17] Lancichinetti, A., Fortunato, S. and Radicchi, F., Benchmark graphs for testing community detection algorithms, Phys. Rev. E 78(4) (2008) 046110.

[18] Lusseau, D., Schneider, K., Boisseau, O. J., Haase, P., Slooten, E. and Dawson, S. M., The bottlenose dolphin community of Doubtful Sound features a large proportion of long-lasting associations, Behav. Ecol. Sociobiol. 54 (2003) 396-405.

[19] Newman, M. E. J. and Girvan, M., Finding and evaluating community structure in networks, Phys. Rev. E 69 (2004) 026113.

[20] Radicchi, F., Castellano, G., Cecconi, F., Loreto, V. and Parisi, D., Defining and identifying communities in networks, J. Proc. Nat. Acad. Sci. USA 101(9) (2004) 2658-2663.

[21] Shen, H. W., Cheng, X. Q. and Fang, B. X., Covariance, correlation matrix and the multiscale community structure of networks, Phys. Rev. E 82 (2010) 016114.

[22] Shen, H. W. and Cheng, X. Q., Uncovering the community structure associated with the diffusion dynamics on networks, Journal of Statistical Mechanics Theory $\& 3$ Experiment 4 (2010) 147-167.

[23] Shen, H. W., Cheng, X. Q., Wang, Y. Z. and Chen, Y. X., A dimensionality reduction framework for detection of multiscale structure in heterogeneous networks, J. Comput. Sci. Technol. 02 (2012) 341-357.

[24] Zhou, H. and Lipowsky, R., Network brownian motion: A new method to measure vertex-vertex proximity and to identify communities and subcommunities, Lect. Notes Comput. Sci. 3038 (2004) 1062-1069. 